\title{
Design investigation to improve voltage swing and bandwidth of the SiGe driver circuit for a silicon electro-optic ring modulator
}

\author{
A. Fatemi ${ }^{1}$, H. Gaul ${ }^{2}$, U. Keil ${ }^{2}$, and H. Klar ${ }^{1}$ \\ ${ }^{1}$ Institute of Microelectronics, Technical University of Berlin, Berlin, Germany \\ ${ }^{2}$ FCI Deutschland GmbH, Berlin, Germany \\ Correspondence to: A. Fatemi (adel.fatemi@tu-berlin.de)
}

Received: 03 December 2014 - Revised: 09 June 2015 - Accepted: 18 June 2015 - Published: 3 November 2015

\begin{abstract}
This paper reports on a new SiGe driver IC to address the low breakdown voltage level of modern BiCMOS transistors. An optical modulator driver IC in SiGe $250 \mathrm{~nm}$ technology with a supply voltage of $4.5 \mathrm{~V}$ is presented. This driver IC consists of pre- and main driver stages where a newly modified cascode topology and capacitance degeneration technique is employed to meet current application requirements; high voltage swing at high datarate. The simulation results show a differential output voltage swing of $3.9 \mathrm{Vp}-\mathrm{p}$ at $14 \mathrm{Gbps}$ data rate, according to the FDR Infiniband standard.
\end{abstract}

\section{Introduction}

In recent years, many studies have worked to solve the bandwidth limitations of wire interconnection and the resulting cross talk in data communication. Photonics could be one way to solve this issue, even at higher transmission distances. By integrating optics into electronics, silicon phonic technology promises to offer a higher level of integrity, greater bandwidth and lower energy consumption in data transmission (Zuffada, 2012).

The optical modulator is a key element to implement the electrical-to-optical data conversion chain on the transmitter side. A micro-ring resonator, fabricated on a silicon substrate, is a high speed optical modulator (Zuffada, 2012). It maps voltage swing across its terminal to a specific level of optical output power. The proper Extinction Ratio (ER) is one of the main quality factors for optical modulation and is therefore dependent on the amplitude of the swing across the ring. Measurement results indicate (Giesecke et al., 2014) that voltage modulation must be in the range of $3-4 \mathrm{~V}$ to provide an acceptable ER. Since a back plane electrical signal could not directly fulfill this requirement (InfiniBand Architecture, 2012), an inter-stage broadband amplifier (here known as a driver IC) is needed to complete the electrical-tooptical conversion chain.

In broadband amplifier, Current Mode Logic (CML) configuration is typically used as the last stage of amplifier. Nowadays, with increasing transient frequency of modern BiCMOS transistors, their breakdown voltage capability is going to lower values (Mandegaran and Hajamiri, 2004). Therefore, obtaining high voltage swing in high transmission data rate is not straightforward.

In Sect. 2 of the present paper, a new modified cascode topology that divides all of the voltage stress among three transistors is explored, and a capacitance degenerative technique that extends bandwidth is also reviewed. Driver IC designed in SiGe $250 \mathrm{~nm}$ technology will be explored in Sect. 3.

\section{Design concept}

\subsection{Modified breakdown voltage doubler of bipolar transistors}

In a CML configuration, the output transistor has to sustain the entire output voltage swing. Cascode topology is a conventional solution to divide voltage stress between two transistors. A number of techniques have been reported to tune the amount of voltage drop on each transistor (Mandegaran and Hajamiri, 2004; Li et al., 2005a, b; Li and Tsai, 2006; Rakowski et al., 2012). All of these techniques are based on pushing the base of the upper transistor, Q2, to follow the output voltage as shown in Fig. 1. 


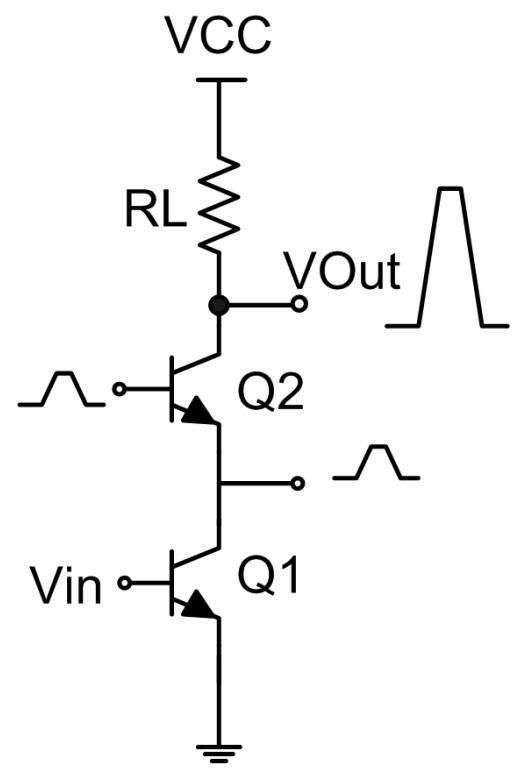

Figure 1. The idea behind modified cascode topology.

If the output voltage is at its maximum, it is desired to evenly divide the stress across two transistors in the case of similar devices in the stacks. A possible solution reported $(\mathrm{Li}$ et al., 2005a) is to use the intrinsic collector-base capacitance (CCB) of the upper transistor, Q2, to feed part of the output signal back to its base. In this way, both the base of the upper transistor and its emitter follow the collector as shown in Fig. 2.

The precise analysis of the feedback network is not straightforward, as the influence of the feedback network and transistor, Q2, must be taken into account as is explained here (Li and Tsai, 2006). The simplified feedback network, with neglecting the effects of large base-collector ohmic resistance and other parasitic capacitances, can be modeled by $\mathrm{C} 1, \mathrm{CCB}, \mathrm{R} 1$, and $\mathrm{R} 2$ as is highlighted in Fig. 3.

A resistive feedback network ( $\mathrm{R} 2$ and $\mathrm{R} 1$ ) defines low frequencies of amplitude voltage of base of transistor, Q2, and $\mathrm{C} 1$ with collector-base capacitance provides voltage drop for higher frequencies. Choosing right value for $\mathrm{C} 1$ is quite important as smaller capacitance brings some spikes at the base of the transistor and higher values of $\mathrm{C} 1$ limits the bandwidth as is shown in Fig. 3. It should be considered that R1 and R2 are chosen comparably larger than RL to reduce power consumption and have less influence on $50 \mathrm{ohm}$ load impedance (Li and Tsai, 2006). Vdc controls the DC voltage of the base and emitter of Q2. Maximum single-ended output swing is calculated using the following equation:

$$
\begin{aligned}
\Delta V_{\mathrm{out}} & =V_{\mathrm{out}, \mathrm{OFF}}-V_{\mathrm{out}, \mathrm{ON}}=\left(2 \times V_{\mathrm{CE}, \mathrm{max}}+V_{\mathrm{CE}, \mathrm{sat}}\right) \\
& -3 \times V_{\mathrm{CE}, \mathrm{sat}} .
\end{aligned}
$$

In the present paper, the idea of using the intrinsic collectorbase capacitance for the feedback network is extended into a

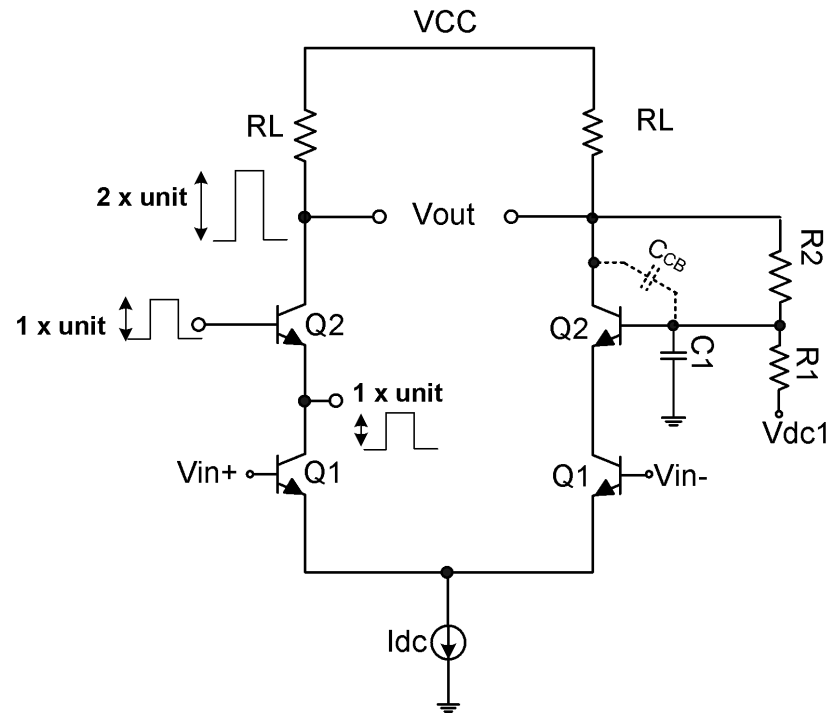

Figure 2. Modified cascode topology with feedback network and proper amplitude voltage ratio.

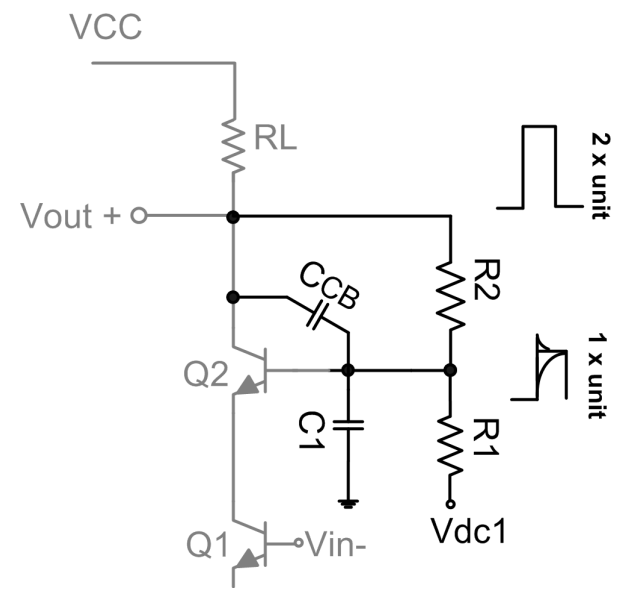

Figure 3. Simplified feedback network in half circuit differential mode.

three-stage cascode topology. This method can increase the maximum allowable output voltage swing while breakdown voltage is becoming less for upcoming downsized technologies. Now, maximum single-ended output swing is calculated using the following equation:

$$
\begin{aligned}
\Delta V_{\text {out }} & =V_{\text {out,OFF }}-V_{\text {out }, \mathrm{ON}}=\left(3 \times V_{\mathrm{CE}, \mathrm{max}}+V_{\mathrm{CE}, \mathrm{sat}}\right) \\
& -4 \times V_{\mathrm{CE}, \mathrm{sat}} .
\end{aligned}
$$

For an identical output swing, it can be proofed that the transistors with lower breakdown voltage, as almost two-thirds of the breakdown voltage in previous method, can be employed.

In the new implementation, the entire output swing must be divided equally among three transistors, so that the base of the two upper transistors must be pushed with the same phase as the output node (collector of Q3) with proper am- 


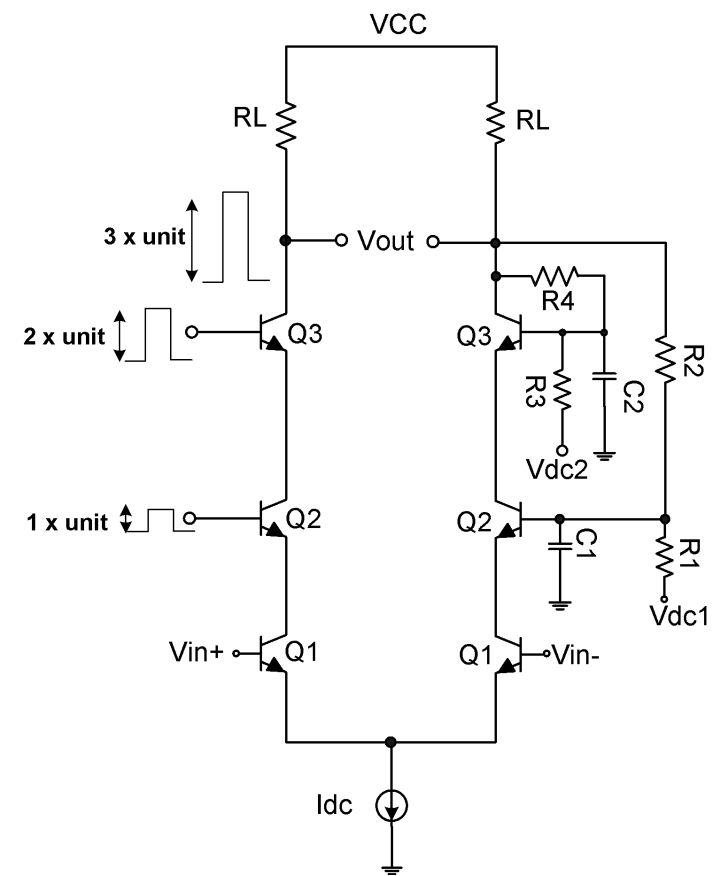

Figure 4. New modified multi-cascode topology with feedback network and corresponding voltage swing.

plitude. As is shown in Fig. 4, the feedback network must convey two-thirds, and one-third of the output signal for the base of Q2 and Q3 respectively, so as to drop one third of the whole output swing over each transistor. Here, the intrinsic collector-base capacitances, together with $\mathrm{C} 1$ and $\mathrm{C} 2$, provide a high frequency feedback network for Q1 and Q2.

\subsection{Capacitance degenerative technique}

In a differential configuration pair, a capacitance degeneration technique could improve bandwidth by adding a pole and a zero to the system (Razavi, 2012). The zero is designed to coincide with and cancel the pole of the system, and the $3 \mathrm{~dB}$ bandwidth is determined by the second pole, which is away from the first one.

Figure 5 shows how the pole and the zero are added to the CML in a differential configuration. For half of the circuit in Fig. 5b, conductance of the common-emitter circuit is (Razavi, 2012):

$G_{m}=\frac{g_{m}}{1+g_{m} \times\left(\frac{R_{s}}{2} \| \frac{1+g_{m} \times \frac{R_{S}}{2}}{R_{S} \times C_{S}}\right)}$.

If one assumes that the output pole of the differential pair is dominated by $R_{L} \times C_{L}$, it would coincide with the added zero to cancel each other out, only if:

$R_{S} \times C_{S}=R_{L} \times C_{L}$.

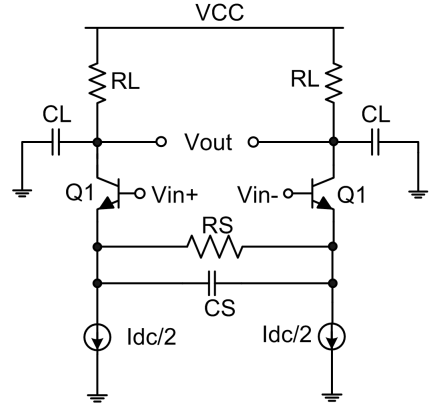

(a)

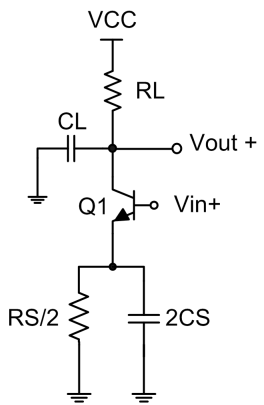

(b)
Figure 5. (a) Capacitance degeneration technique, (b) half circuit in differential mode.

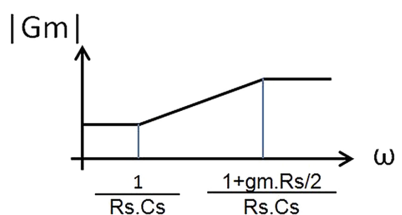

(a)

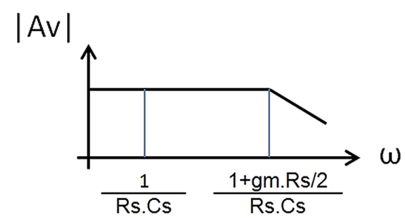

(b)
Figure 6. (a) Bandwidth of Gm, (b) overall system frequency response.

Therefore, overall bandwidth of the system is extended to the second pole of the system, $\left(1+g_{m} \times R_{S} / 2\right) /\left(R_{S} \times C_{S}\right)$, as shown in Fig. 6.

\section{Design and implementation}

Using the concepts discussed in Sect. 2, a driver IC was implemented in SiGe $250 \mathrm{~nm}$ technology. This driver IC consists of two parts: a pre-driver stage and a main driver stage.

\subsection{Pre-driver stage}

Back-plane electrical signals fall within a certain standard range. This range is normally not strong enough to completely switch the current from one differential side to another one, a requirement for maximizing output swing and minimizing power consumption. In addition, a high amplitude input signal could bring the transistors into a deep saturation region where switching speed is reduced. Therefore, the pre-driver stage is placed before the main driver stage in order to prepare both the amplitude and the DC level of the input signal for the main stage driver IC.

As is shown in Fig. 7, the pre-driver stage is in a differential configuration, which consists of one commonemitter amplifier, followed by a common-collector stage. The common-emitter stage is designed to transfer low and high amplitude input signal to the specific level appropriate for driving the main stage driver IC. The main role of the common-collector stage is to provide proper impedance 


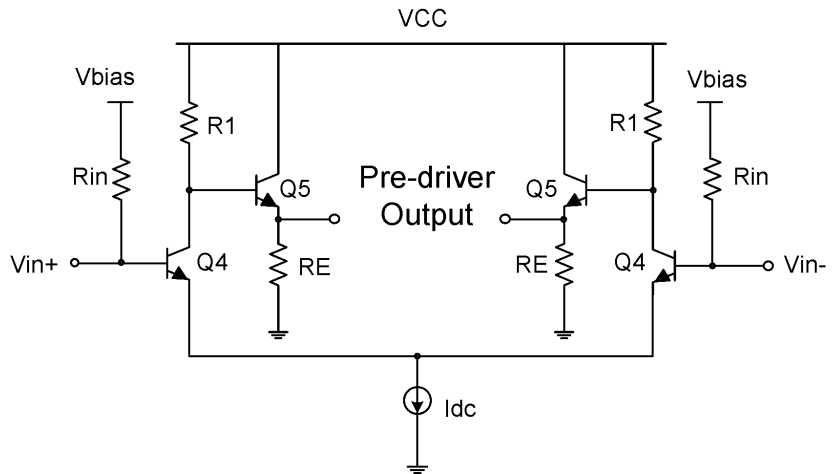

Figure 7. Proposed pre-driver stage.

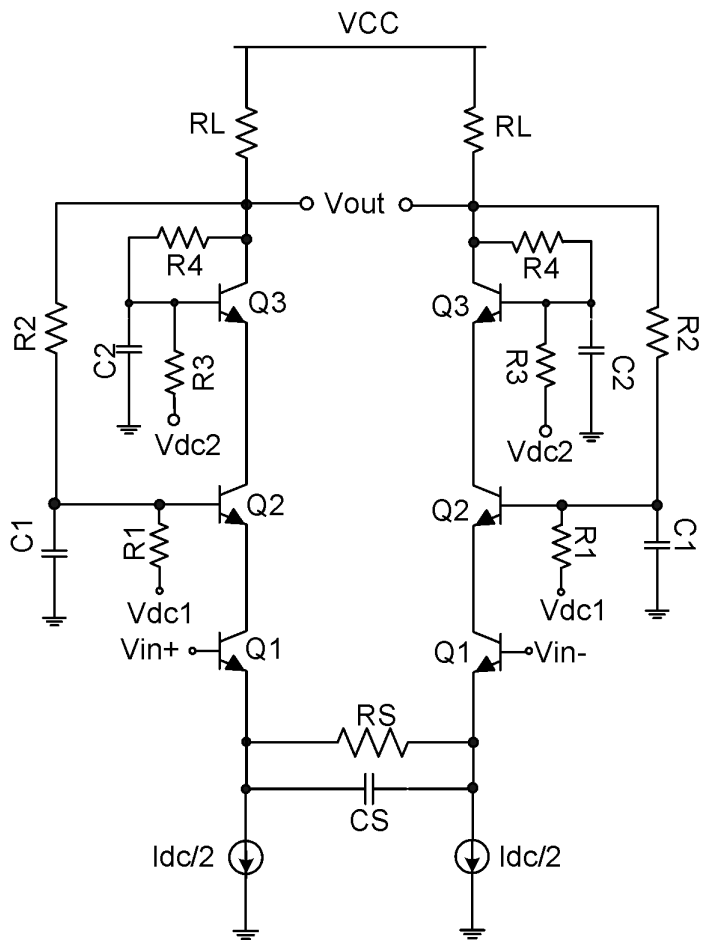

Figure 8. Proposed three stage cascode configuration.

for the common-emitter amplifier and cascade stage. In the present paper, the pre-driver output is designed to generate $500 \mathrm{mVp}$-p for the main driver stage.

\subsection{Main driver stage}

As target differential output swing is $4 \mathrm{Vp}-\mathrm{p}$, supply voltage is increased to $4.5 \mathrm{~V}$. Figure 8 shows how the main stage driver IC is constructed, it consists of a three-stage cascode and capacitance degeneration technique. A simulated eye diagram is depicted in Fig. 9, where its eye opening is $3.9 \mathrm{~V}$ at 14 Gbps.

Figure 10 shows how much voltage stress is on each device. This figure indicates that stress is less than $1.5 \mathrm{~V}$, which

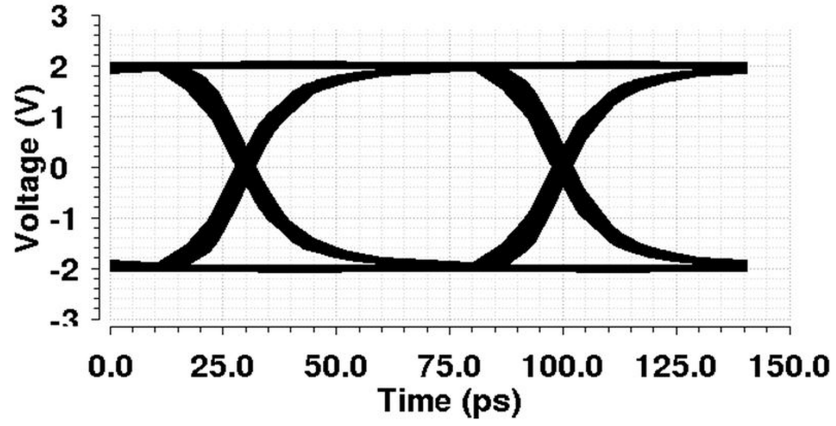

Figure 9. Simulated eye diagram of output signal at $14 \mathrm{Gpbs}$.

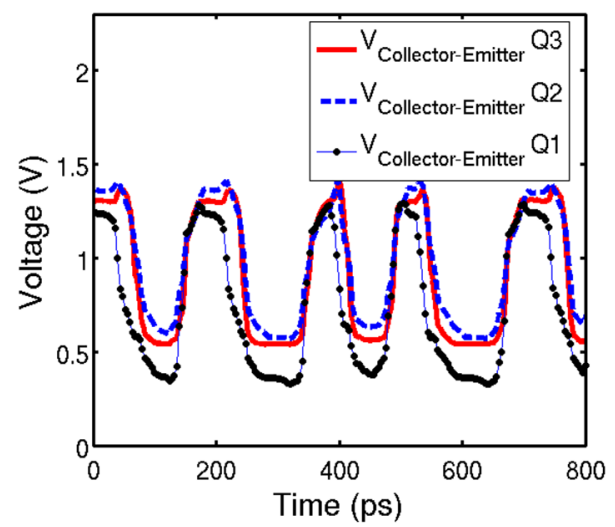

Figure 10. Simulated collector-emitter voltage stress in multicascode topology.

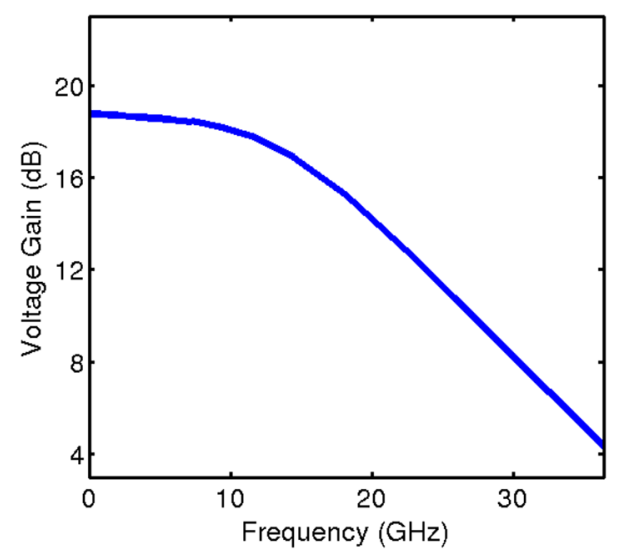

Figure 11. Simulated small signal voltage gain of driver IC.

is less than the breakdown voltage of $1.9 \mathrm{~V}$ in this technology, and it is shared quite evenly among devices as well. Figure 11 shows the small signal simulation of driver IC that indicates low frequency gain of $17 \mathrm{~dB}$ and corresponding $3 \mathrm{~dB}$ bandwidth of $14 \mathrm{GHz}$. 


\section{Conclusions}

Silicon photonic technology offers a way to fully integrate optics into electronics in a single chip. A micro-ring resonator requires a comparatively high input voltage swing for proper performance (minimum ER). In the present paper, some techniques have been presented to obtain a high voltage swing in multi-Gbps data rate transmission. The technique that is introduced in this paper makes it possible to employ the transistors with lower breakdown voltage levels as twothird for high voltage swing application. Finally, the SiGe driver IC in $250 \mathrm{~nm}$ technology was investigated. Simulation results claim an output voltage swing of $3.9 \mathrm{Vp}$-p at $14 \mathrm{Gbps}$.

Acknowledgements. This work is funded by the Federal ministry of education (BMBF) in the SHyWA project under Grant No. 16BP1103. The Author would like to thank Friedel Gerfers for his valuable discussions and guidance.

Edited by: D. Killat

Reviewed by: two anonymous referees

\section{References}

Giesecke, A. L., Prinzen, A., Bolten, J., Porschatis, C., Chmielak, B., Matheisen, C., Wahlbrink, T., Lerch, H., Waldow, M., and Kurz, H.: Add-drop microring resonator for electro-optical switching and optical power monitoring, in: Proceedings of the 34th Conference on Lasers and Electro-Optics, 08-13 June 2014, San Jose, USA, 1-2, 2014.

InfiniBand Architecture, Volume 2: https://cw.infinibandta.org/ document/d1/7141, last access: November 2012.

Li, D. U. and Tsai, C. M.: 10-Gbps modulator drivers with local feedback networks, IEEE J. Solid-St. Circ., 1, 1025-1030, 2006.

Li, D. U., Haung, L. R., and Tsai, C. M.: Low power consumption 10-Gbps SiGe modulator drivers with $9 \mathrm{~V}_{\mathrm{PP}}$ differential output swing using intrinsic collector base capacitance feedback network, IEEE Rad. Freq. Integr., 1, 317-320, 2005 a.

Li, D. U., Haung, L. R., and Tsai, C. M.: 10 Gbps CMOS laser driver with $3.3 \mathrm{~V}$ output swing, IEEE Cust. Integr. Cir., 1, 333 336, 2005 b.

Mandegaran, S. and Hajimiri, A.: A breakdown voltage doubler for high voltage swing drivers, IEEE Cust. Integr. Cir., 1, 103-106, 2004.

Rakowski, M., Ryckaert, J., Pantouvaki, M., Yu, H., Bogaerts, W., De Meyer, K., Steyaert, M., Absil, P. P., and Van Campenhout, J.: Low power, 10-Gbps 1.5-Vpp differential CMOS driver for a silicon electro-optic ring modulator, IEEE Cust. Integr. Cir., 1, 9-12, 2012.

Razavi, B.: Capacitance Degeneration, in: Design of Integrated Circuit for Optical Communications, Second Edition, John Wiley and Sons, Hoboken, New Jersey, 140-143, 2012.

Zuffada, M.: The industrialization of the Silicon Photonics: Technology road map and applications, in: Proceedings of the 42th European Solid-State Device Research Conference, 1721 September 2012, Bordeaux, France, 7-13, 2012. 